\title{
STRATÉGIE ET BONDAGE DU GÉNÉRIQUE : SOCIO-SÉMIOTIQUE DE LA SÉRIALITÉ AUDIOVISUELLE
}

Claudia Attimonelli

De Boeck Supérieur | «Sociétés »

2015/2 n 128 | pages 9 à 18

ISSN 0765-3697

ISBN 9782807301337

Article disponible en ligne à l'adresse :

http://www.cairn.info/revue-societes-2015-2-page-9.htm

\section{Pour citer cet article :}

Claudia Attimonelli, «Stratégie et bondage du générique : socio-sémiotique de la sérialité audiovisuelle », Sociétés 2015/2 ( $\left.n^{\circ} 128\right)$, p. 9-18.

DOI 10.3917/soc.128.0009

Distribution électronique Cairn.info pour De Boeck Supérieur.

(C) De Boeck Supérieur. Tous droits réservés pour tous pays.

La reproduction ou représentation de cet article, notamment par photocopie, n'est autorisée que dans les limites des conditions générales d'utilisation du site ou, le cas échéant, des conditions générales de la licence souscrite par votre établissement. Toute autre reproduction ou représentation, en tout ou partie, sous quelque forme et de quelque manière que ce soit, est interdite sauf accord préalable et écrit de l'éditeur, en dehors des cas prévus par la législation en vigueur en France. Il est précisé que son stockage dans une base de données est également interdit. 


\section{STRATÉGIE ET BONDAGE DU GÉNÉRIQUE : SOCIO-SÉMIOTIQUE DE LA SÉRIALITÉ AUDIOVISUELLE}

Claudia ATTIMONELLI

« Mère, donne-moi le soleil. » Henrik Ibsen, Les Revenants.

Pour activer la pratique de la vision capable de faire émerger de la réalité des univers fantastiques, il est avant tout nécessaire de stipuler avec nous, les spectateurs, un contrat de confiance selon lequel - de manière systématique et à cadence fixe - nous croirons à un monde imaginaire en échange de la promesse d'être catapultés dans un lieu et un temps vraisemblables pendant toute la durée de l'épisode. Un tel contrat de véridiction nous suspendra dans une langueur proche du plaisir jusqu'à l'avènement de l'épisode suivant. Ce dernier, autrefois attendu avec ferveur conformément à la scansion du temps structurée par la traditionnelle grille des programmes, est désormais avidement consommé aussitôt après le précédent; il arrive même qu'il soit ensuite ruminé, que certaines de ses scènes soient reprises et se transforment en fétiches médiatiques et emblématiques que nous pouvons citer, revivre, relier aux hyperdiscours dont se nourrit notre mondanité quotidienne : ainsi deviennent-elles des scènes culte. En d'autres termes, les fans se retrouvent dans l'univers sociétal que le télé-alchimiste a créé pour eux, dont ils reconnaissent les anfractuosités, se rappellent les trames parallèles et citent les détails. Ils peuvent alors jouir des imperfections issues de l'écart entre la scène culte et le monde des TV serials. Eco, qui voit ces imperfections comme des ornements déterminants dont les fans peuvent extraire des lambeaux à déchiqueter et exhiber comme fétiches cultuels, les qualifie de "frames magiques ${ }^{1}$ capables de déclencher le dispositif du déjà-vu en convoquant instantanément le spectateur

* Sociosémiologue, docteur en théorie du langage et sciences des signes, est chercheuse à l'Université Aldo Moro de Bari, où elle enseigne cinéma, photographie et télévision.

1. Cf. U. Eco, Travels in Hyperreality, Harcourt, New York, 1986. 
face au miroir des multiples mondanités où il a déjà perçu, vu, senti et vécu sur sa propre peau - ou empathiquement effleuré sur la peau des autres - un geste, une boutade, une moue, une combinaison de posture et de nuance, une modalité de réaction et d'approche des événements quotidiens. Les frames magiques fondent le culte d'une série ${ }^{2}$. Il y a par exemple la «mort par eau » de Charlie, sur la paume duquel Desmond découvre l'inscription « NOT PENNY'S BOAT »; l'événement médiatique de 1980, "Qui a tiré sur J.R. ? » (Dallas), qui devient un élément intrasémiotique migrant entre divers lieux télévisuels, de Friends à The Simpsons ; la chute de Carrie vêtue d'une tenue époustouflante lors de son premier défilé en tant que non-modèle pour Dolce \& Gabbana ; la première scène d'amour passionnée entre Buffy et Spike, exacerbée par une longue tension érotique (en ce cas, le culte s'étend en ligne jusqu'au téléchargement de la part des fans du finale censuré de cet épisode) ; ou encore, les phrases annonçant l'apparition du nain et de Laura Palmer dans la chambre rouge de la minisérie Twin Peaks : "Dans l'obscurité d'un futur passé / Le mage désire voir / Un homme chante une chanson entre ce monde et l'autre / Feu marche avec moi. » Les exploits des protagonistes sériels se transforment en scènes de culte par le biais d'un prélèvement vampirique qui ré/génère le lien entre le spectateur (désormais fan) et le héros.

À côté de cette transformation, l'autre élément intrinsèque de la série à succès est la construction du mythe. Ici le langage sériel se fait métalangage, message avant-coureur de contenus dépassant le sens de l'intrigue. Ainsi se définissent ces ingrédients - des traits distinctifs uniques pour chaque série - qui arrachent le message au quotidien ordinaire pour le plonger dans le magique, le surnaturel, le loufoque, l'excédent, le grotesque : autrement dit, qui créent des mythes. Dans Mythes et rites d'aujourd'hui $(1977)^{3}$, Dorfles consacre un chapitre à la constitution du mythe de la SF, qui partage certains critères avec l'essence du mythe dans le phénomène des TV serials, dont : la vraisemblance, la donnée scientifique, l'improbabilité, le regard sur les milieux linguistique, publicitaire, cyborg, anthropologique, biopolitique, ainsi que l'adoption de jargons spécialisés, bien entendu.

Per lumina, en analogie avec certains éléments cultuels précédemment décrits, les mythes sériels correspondants pourraient être : J.R. s'approchant, avec sa démarche texane, du mobile-bar pour se préparer un drink ; Carrie qui, le soir, écrit à l'ordinateur son morceau du lendemain, allongée sur son lit, ses orteils ondoyant derrière sa crinière léonine; les terribles coups de pied et de poing de Buffy en ouverture de chaque épisode, qui dénotent surtout le conflit typique de l'héroïne des années 1990 tiraillée entre force physique/sauvetage-du-monde/ carrière et abandon aux plaisirs charnels et à la normalité d'une histoire d'amour ; parmi les mythes sériels, mentionnons aussi les oreilles thériomorphiques de Spock associées aux sourcils froncés et à la teinte céruléenne de l'uniforme; la posture

2. Cf. L. Spaziante, «TV sui generis: strategie di presa di distanza e generi in Twin Peaks », in G. Grignaffini, M. P. Pozzato (dir.), Mondi seriali. Percorsi semiotici nella fiction, Collana Link, RTI-Reti Televisive It, Cologno Monzese, 2008.

3. Cf. G. Dorfles, Nuovi riti, nuovi miti (1965), Einaudi, Torino, 1977. 
légèrement bossue d'Horatio éclairé par le coucher de soleil orange sur Miami ; l'arrogance, mitigée par une tension croissante, perturbante et irrésolue, de Tony Soprano en séance avec son analyste Jennifer Melfi.

Lorsque la textualité sérielle donne lieu à la constitution de mythes modernes, ces derniers, de par leur nature médiatique, deviennent aussitôt des fragments migrants susceptibles de se traduire dans tout autre contexte extérieur à la série elle-même ; autrement dit, ils constituent un fonds d'archives diurnes dans lequel nous puisons quotidiennement et un plan d'intensité nocturne auquel nous cédons en un jeu érotique.

Les formes de consommation passionnée, empathique, collective ou solitaire, ne sont pas seulement dues au plaisir du retour sériel d'un méta- et pluri-vers auquel nous avons décidé de nous adonner ; elles découlent surtout de la signature exprimant notre consentement à l'abandon d'un rôle et l'adoption d'un autre quand, assis ou semi-allongés, face à la surface plane de nos écrans, nous nous préparons à la vision de la répétitivité fictionnelle élevée au plus haut niveau de jouissance sensorielle. Et tout cela se produit en vertu de certaines stratégies énonciatives, narratives et de marketing impliquant la syntaxe entière des séries télé, du générique de début à celui de fin - lequel est systématiquement coupé. À cet égard, il est intéressant de reconsidérer l'effet de distanciation provoqué par l'interruption ex abrupto de l'épisode sur les premières notes du générique de fin, où se concentrent d'ordinaire toutes les informations et crédits que le générique de début n'est pas tenu d'énoncer ; c'est là que le spectateur est brutalement ramené à la réalité après avoir été conduit au sommet de la tension narrative grâce à la stratégie du cliffhanger, qui, dans la pratique du fétiche, constituerait le principe essentiel et la règle du jeu. Le cliffhanger est le suspense, qui suspend justement le spectateur à l'acmé de la tension et l'incite à visionner l'épisode suivant.

\title{
Le générique comme seuil vers le Pleasuredome
}

\author{
"Quand viendra le matin livide, \\ Tu trouveras ma place vide. \\ où jusqu'au soir il fera froid. » \\ Charles Baudelaire, Le Revenant.
}

Dans la pratique de la lecture individuelle, hormis le bref rituel nous introduisant au texte chaque fois que nous le reprenons en main, que ce soit l'installation dans notre fauteuil préféré, les jambes en position confortable, ou la création d'un micro-espace dans le siège de l'avion qui accompagnera notre envol vers les mondes représentés, il n'existe pas d'introduction animée à l'œuvre, sauf si nous entendons par là la page de garde, le frontispice ou le colophon avec les signes des gens pour lesquels le livre a été imprimé. De fait, l'histoire se remet à vivre à travers notre lecture sans générique préalable. Celui-ci est en effet propre au langage audiovisuel. Il se déploie selon une ponctuation textuelle et paratextuelle fort 
complexe : sur le plan syntagmatique, c'est le « à la ligne ${ }^{4}$ de la programmation télévisuelle, qui sépare l'avant de l'après et introduit un nouveau contenu; sur le plan symbolique, c'est l'approche du lieu où nous obtiendrons notre dose de plaisir, comme si, chaque fois que nous nous disposons à visionner une série qui nous intéresse, nous parcourions avec un frisson d'excitation ce bref segment du sentier désormais battu qui conduit jusqu'à l'entrée, nous arrêtions sur le seuil, ouvrions la porte en frémissant de curiosité, et nous installions commodément pour mieux nous propulser dans l'univers de nos héros ${ }^{5}$. Tel est le générique : préparation, ritualité, tension, imagination, allusion, abandon, suspension, répétition.

Pour avancer une reconstruction tenant compte des usages et des pratiques, on pourrait dire que le générique d'ouverture des séries véhicule de nombreux signifiés, dont la séduction du spectateur, l'allusion aux contenus du texte à venir, la communication de l'identité par le biais du logo ${ }^{6}$, la mention des crédits de ceux qui ont participé à la création du produit, la dissémination de segments de valeurs, de tropes et d'icônes activés en vue d'être éventuellement repris par la suite dans le texte filmique. Il se présente sémiotiquement comme moment « d'ouverture d'un contrat communicatif [...], positionnement du spectateur sur un axe de lecture privilégié $\gg^{7}$.

Certains génériques parviennent à instaurer cette relation au moyen de stratégies énonciatives explicitant de façon synthétique les isotopies valorielles et pathémiques dans la brève séquence d'introduction à la série, isotopies susceptibles d'être développées au cours de l'épisode suivant mais quoi qu'il en soit annonciatrices du genre et des topics traités dans la saison en cours. Il s'agit de génériques métonymiques $^{8}$ en tant qu'ils exploitent largement des contenus tirés des épisodes (héros, scènes, éléments et sons) dans un montage rapide qui contribue à créer l'iconographie du mythe sériel (il suffit de penser aux génériques de Buffy the Vampire Slayer, Smalville, Lost, Dallas, Friends). Les premiers plans, le zoom sur les visages avec

4. Cf. F. Cicolani, Teoria e tecnica della sigla televisiva, il paratesto televisivo e la sua applicazione pratica, Tesi di laurea, 2005.

5. Cf. C. Attimonelli, A. D'Ottavio, To Be Continued. Il destino del corpo nei serial televisivi, CaratteriMobili, Bari, 2011.

6. À cet égard, il est utile de rappeler que le passage désormais imminent de nombreux $T V$ serials au web, avec ce que cela implique de révision des temps et des modes d'utilisation, entraînera avant tout la suppression du générique, ce qui en fera un objet de culte, comme ceux des téléfilms du passé. Cette perspective effraie déjà nombre de fans, à l'heure où bien des épisodes de séries à succès transmises à la télévision ont déjà perdu leur opening sequence ; on peut en effet lire dans un forum : " J'ai remarqué ces derniers temps que les génériques des TV serials ont disparu en faveur du seul logo de la série. Voir Jericho, Lost, Grey's anatomy, Supernatural, Heroes, etc. Ils faisaient partie intégrante de l'épisode et rendaient la série plus palpitante. »

7. N. Dusi, «La variante interna. Modularità e ripetizione nell'epitesto audiovisivo ", in Mutazioni audiovisive, ETS, 2004, p. 3.

8. F. Cicolani, Teoria e tecnica della sigla televisiva, il paratesto televisivo e la sua applicazione pratica, op. cit. 
effets de lumière, les poses bizarres ${ }^{9}$, tels les coups de pied de Buffy ou l'envol de Superman, servent à fixer icastiquement, en les exaspérant jusqu'au grotesque, certains traits distinctifs sur lesquels se moduleront les visions des épisodes, afin qu'ils restent imprimés dans l'imaginaire quotidien du spectateur et résolvent l'apparente contradiction entre vraisemblance et mythographie.

Une autre série recourant à la citation des épisodes est Friends, où les protagonistes bien-aimés, présentés dans les situations typiques, n'en font qu'un avec leur expression typique, idiote, sarcastique, surprise, tendre ou vexée, éléments symboliquement réunis sur le divan en cuir qui, au cours des saisons suivantes, deviendra prépondérant dans la construction du générique lui-même ${ }^{10}$.

Ladoption d'écritures co-adjuvantes constitue un moyen encore plus efficace de désigner les isotopies pathémiques et valorielles en introduisant les divers plans auquel le texte filmique se référera au cours de la saison. Les notes initiales du générique de X-Files, par comparaison avec la fanfare de Star Trek, évoquent l'élément de science-fiction ramené sur terre et mêlé à notre quotidien, donc précisément perturbant parce que potentiellement réel. D'autres composantes interviennent pour recréer ce milieu liminal et corroborer sa scientificité : ce sont les mots présents sur les écrans et sur les cartes des agents spéciaux Molder et Skully, qui acquièrent par défaut, en vertu de leur nature de document, la véridicité inhérente à la donnée scientifique. Paranormal activity, Agent of investigation FBI, Government denies knowledge, The truth is out there, ainsi se construit l'architecture à partir de laquelle se déploieront les plans d'intensité du produit sériel, à savoir l'enquête sur le paranormal, le fait que celle-ci soit menée par des agents spéciaux et non de simples policiers, le caractère secret des investigations, l'implication du gouvernement et, pour finir, le payoff renvoyant à une vérité toute prête qui nous attend là, à l'extérieur - ou plus exactement là, dans l'écran.

\section{Ostension chirurgicale et dissimulation sonique du corps dans la pratique du générique}

"Join the Corpse", Sidney Zombie Walk.

Les génériques qui marquent la mémoire du public pendant des décennies sont ceux qu'on pourrait qualifier de métaphoriques, dans le sens où ils font appel au pouvoir allusif de symboles et d'icônes, d'apparitions de mots, de graphèmes et de stratégies énonciatives éminemment évocatrices. Ce type de générique colle le spectateur à l'écran en suggérant sans résoudre l'énigme, il l'attache au divan du plaisir sériel avant même la diffusion effective du TV serial, et ne le libère que lorsque les notes accompagnant le générique de fin interrompent la tension sensuelle.

9. Cf. R. Barthes, Système de la mode, Éditions du Seuil, Paris, 1967.

10. Il est intéressant d'évoquer un autre célèbre divan présent dans un générique sériel, celui des Simpsons, sur lequel, après des péripéties chaque fois différentes et rocambolesques, les membres de la famille se retrouvent assis à la fin du clip, prêts à se regarder eux-mêmes à la télévision. 
À partir de la saison The new generation de Star Trek, diffusée en Italie de 1991 à 1997 (quatre ans plus tard qu'aux États-Unis), une relation privilégiée avec l'élément sonore s'instaure. Dans les sigles métaphoriques, la musique matérialise le texte et finit par coïncider aussi avec sa forme : la bande-son du générique de Star Trek, composée par Alexander Courage, est tout simplement mémorable.

Ici, l'effet final est produit par deux éléments : l'absence de figures humaines remplacées par l'astronef Enterprise plongé dans la lumière noire de l'espace, tandis qu'il survole Saturne, et la voix extradiégétique du célèbre prologue : «Lespace, l'ultime frontière, voici le vaisseau galactique Enterprise. Sa mission : explorer des mondes nouveaux et étranges, découvrir de nouvelles formes de vie et de nouvelles civilisations, et s'aventurer dans les recoins les plus éloignés de la galaxie ${ }^{11}$. »

Les notes d'Angelo Badalamenti, qui forment le tapis du générique de Twin Peaks, s'animent sur une vague sonore que le théoricien de l'afro-futurisme Kodwo Eshun définirait comme sonifiction. Le symbolisme magique des lieux et l'absence d'êtres humains, en ce cas, s'avèrent particulièrement efficaces pour évoquer les arcanes de Lynch. Nous pouvons identifier quatre figures essentielles qui véhiculent le vide d'informations concernant le meurtre de Laura Palmer : l'oiseau en ouverture, porteur d'un mystérieux message, le long plan sur la fraiseuse en train de façonner les dents d'une roue dentée, le panneau signalant l'entrée à Twin Peaks, la cascade et la rivière où nagent deux volatiles, emblème du lieu où l'on découvre le cadavre enveloppé de plastique. En d'autres termes, le vide apparent autour duquel tourne la longue enquête de l'agent spécial Cooper.

Le stratagème consistant à ne citer que certains des niveaux du texte sériel dans le générique - en phase avec les pratiques fétichistes qui effectuent des prélèvements de la partie en violant et omettant le tout, dont elles n'ont que faire - peut en outre rendre ce langage très semblable aux vidéoclips musicaux privilégiant une certaine distanciation du texte visuel par rapport au texte musical. Par exemple, le générique de Nip/Tuck, tourné dans un parfait style vidéoclip, souligne de manière trop didactique les thèmes développés par la série, s'appuyant sur une succession d'images et une chanson dont voici les paroles : « Make me beautiful / perfect soul / perfect mind / perfect face / a perfect lie. » Ici, la proximité entre texte filmique et bande-son, dont l'un est la traduction servile de l'autre, maintient très bas le niveau iconique - autrement dit l'ouverture à une pluralité d'interprétations, qui sauve une cuvre et la présélectionne pour l'imaginaire du futur.

11. La première génération des pionniers de la Techno de Detroit a utilisé des segments de ce prologue, telle la fameuse phrase volontiers citée lors des interviews par Derrick May, qui soutenait que la musique techno explorait de nouveaux mondes et entendait « aller là où personne n'est encore arrivé ». Hustler, la maison de production de films X, effectua quant à elle une opération citationniste radicalement différente, en altérant légèrement le texte, lorsqu'elle réalisa le remake porno de Star Trek : « [...] to arrive again and again where no man has come before » (jeu de mots sur le verbe « come » qui peut signifier « venir » ou « jouir »). 
Le très bref générique de Grey's Anatomy - quelques secondes à peine - représente un bon compromis entre dissémination d'isotopies valorielles et distanciation par rapport à la descriptivité des contenus des épisodes, grâce à la mise en œuvre de certaines stratégies énonciatives efficaces, dont le choix d'introduire des êtres humains qu'on ne peut identifier avec les héros, car seuls des fragments de corps sont montrés : dos, doigts, pieds, œil, tous saisis en train d'effectuer une action se prêtant au moins à deux interprétations. Les bistouris et instruments chirurgicaux de la deuxième scène, alignés par des mains soignées, laissent place en fondu enchaîné au recourbe-cils barrant l'œil au moment du maquillage, hommage à la scène d'ouverture du Chien andalou, le premier film surréaliste réalisé par Dali et Buñuel en 1929. La même technique de raccord introduit ensuite les gestes croisés de mains d'homme remontant la fermeture éclair d'une robe noire et de mains de femme nouant les lacets d'une blouse opératoire sur le dos d'un homme. Puis une table d'opération filmée en plongée est remplacée par des tables de restaurant. Le duo des isotopies continue avec la canule d'une intraveineuse ; le liquide s'écoulant à l'intérieur, dans la neuvième scène, se transforme en jet de champagne tombant dans un verre. Le finale montre des jambes et des pieds entrelacés sur un lit d'hôpital où un couple est en train de faire l'amour, avant de cadrer la paire d'escarpins en satin rouge qu'on avait aperçus, lors de la première scène, parmi d'autres chaussures alignées dans le vestibule des médecins. On a carrément l'impression que le générique de Grey's Anatomy est sous-tendu par un récit que le spectateur, de vision en vision, pourra reconstruire en donnant des visages et des noms aux corps mis en scène.

\section{Narrations génériques : sexe, crime, péché et folie}

"- Elle était morte, et puis hop, elle ressuscite?

[...] Tu comptes la cacher? Mais, c'est dommage. II faudrait faire une grande fête pour son retour ! 》

Les Revenants (SIE2).

Si elle est bien tissée à la trame, la tension narrative annoncée dès le segment filmique du générique d'un TV serial peut constituer une excellente stratégie de séduction. Citons trois cas d'accroche captivante. Le premier est le générique de Sex and the City : Carrie, flâneuse Newyorkaise vêtue d'un tutu rose, traverse avec légèreté la circulation chaotique de la ville en toile de fond (gratte-ciel, ponts, ciel diurne et nocturne). Kiddult avertie, elle sourit et semble complice du milieu qu'elle arpente avec familiarité. Vers la fin, la fable métropolitaine est souillée par une éclaboussure lorsqu'un bus roule dans une flaque, maculant la tenue de Carrie mais attirant aussi son attention déconcertée sur sa propre image en version publicitaire affichée le long du moyen de transport : elle se voit dans une pose sensuelle entre les draps d'un lit, allongée sur le côté en négligé rose. Avec le payoff, "Carrie Bradshaw knows good sex* ", l'élément déjà annoncé par le titre de la série se renforce, entre autres parce qu'un astérisque renvoie à la ligne finale de la pub, où il est spécifié que cette experte du sexe bien fait n'a aucun problème à le demander! 
On retrouve ici, concentrée dans l'isotopie vestimentaire du tutu et du négligé, en quelques fragments dérivés, toute l'histoire des quatre filles de New York.

Dexter et The Sopranos représentent deux cas différents mais complémentaires en termes de développement narratif du générique. Ce qui rapproche ces deux produits, c'est entre autres le trend qui a caractérisé la production sérielle des années 1990, autrement dit la tendance à explorer les ombres et lumières de figures borderline de policiers et de criminels. Tant Dexter Morgan que Tony Soprano incarnent des personnalités déchirées par des pulsions complexes, analysées en profondeur au fil du développement des saisons, en particulier sur le plan psychanalytique. Dans le générique, ce niveau se perçoit par moments, de manière ambiguë : Dexter est filmé peu après son réveil, en train de se livrer à sa routine matinale avant de sortir. Quant à Tony Soprano, au volant de sa voiture, on le suit le long du trajet qui le ramène chez lui, sur fond musical : "You woke up this morning / Got yourself a gun / Mama always said you'd be / The Chosen One / She said: You're one in a million / You've got to burn to shine / But you were born under a bad sign / With a blue moon in your eyes. "Lélément de sensualité criminelle de Tony, ses troubles de la personnalité et sa position d'élection au sein de la communauté dans laquelle il vit sont explicitement énoncés par la bande-son et suggérés par certains tropes cinématographiques désormais mythifiés. Son assurance est d'emblée soulignée par la façon dont il se saisit de la carte au passage du péage, dont il tient son cigare - qu'on peut rapprocher de l'archétype du Parrain -, par le bracelet et la montre en or à ses poignets. Tout ce bassin signique, concentré en quelques photogrammes, est synthétisé par le logo final, où le « $\mathrm{R}$ » central du mot se transforme par alchimie en pistolet au canon tourné vers le bas. Quant aux allusions présentes dans la description de la routine matinale du jeune criminel Dexter, elles sont d'un grand effet visuel. Le générique exploite la décontextualisation d'objets, d'actes et de morceaux de chair humaine et animale, qu'il dissèque en n'en montrant (de façon pornographique) que des détails perçus par le spectateur comme isotopies "souches » permettant de comprendre les com/pulsions violentes et homicides du protagoniste. La profondeur des apparences structure la stratégie énonciative d'un générique qui a le mérite de révéler la nature technologiquement complexe du réel, ici ramenée à la personnalité perturbée d'un killer hématologue.

Un dernier regard sur la tension sérielle sera consacré aux génériques qui exploitent la stratégie énonciative des animations, désincarnant pour l'occasion les personnages et les remplaçant par des avatars digitaux. Deux exemples emblématiques sont les séquences d'ouverture de Desperate housewives et de Mad Men.

La première série opte pour le citationnisme artistique d'œuvres anciennes ou contemporaines, dans un remake ironique et scandaleux des conditions et du statut des protagonistes : on part de la figure d'Ève dont Cranach représenta la sensualité nordique, séraphique et spontanée ; elle s'apprête à offrir une pomme à Adam, mais un autre fruit surdimensionné tombe sur lui et le fait disparaître sous terre. Leur succèdent Néfertari, entourée d'enfants agités, puis les époux Arnolfini (en attente d'un heureux événement) du tableau de Van Eyck, dont 
les occupations domestiques sont perturbées par l'intrusion d'une banane pop. Un zoom nous éloigne du foyer et nous installe devant l'entrée de la maison du couple symbolisant le bigotisme américain bien-pensant, peint par Grant Wood dans American Gothic. C'est alors qu'une pin-up des années 1950 pointe la tête, aguichant le mari sévère tandis que l'épouse de ce dernier est scellée dans une boîte de sardines de la même époque avant d'être déposée sur le plan de travail d'une cuisine. Apparait une heureuse ménagère de l'époque du boom, aux bras si chargés de courses qu'une conserve de tomates Campbell s'en échappe. Elle est rattrapée par un homme dessiné par Liechtenstein, face à sa dulcinée en larmes, laquelle finit par lui envoyer un coup de poing dans l'œil, que l'artiste américain n'aurait jamais conçu.

Le générique de Mad Men est bien plus sobre par rapport à cette chevauchée excentrique et maximaliste sur les sentiers de l'histoire de l'art ${ }^{12}$. Situé dans les années 1960 à l'époque de la campagne présidentielle de Kennedy et Nixon, ce $T V$ serial devenu culte en un temps record, raconte la complexité, les intrigues, les stéréotypes et les bouleversements d'une société américaine à travers le prisme de la vie d'un groupe de publicitaires travaillant pour l'agence Sterling Cooper. La opening sequence est centrée sur la silhouette en costume noir du créatif Don Draper - inspiré du creative head de l'agence Leo Burnett - au moment où celui-ci entre dans son bureau, un des lieux emblématiques de la série. Après une pause, le protagoniste sent soudain le sol se dérober sous ses pieds, au ralenti. Les murs s'effritent, les meubles de design se déstructurent et l'homme tombe en chute libre, mais lentement et comme une plume, d'une hauteur incommensurable qui lui permet de regarder au passage des icônes publicitaires de l'imaginaire quotidien, véritable condensé d'american visual culture rappelant les thèmes traités par la série : la guerre des sexes, les femmes épinglées dans leur nature de femmes fatales et d'anges du foyer, le pouvoir mis en acte par le vêtement, la séduction d'une paire de chevilles féminines qui semblent écarter d'un coup de pied la promesse matrimoniale synthétisée par deux mains et une alliance. Cependant, la chute n'est pas mortelle, et Draper atterrit même confortablement assis sur un divan : on le voit de dos, en train de fumer et d'observer le spectacle sociétal du public plongé dans une attente rêveuse et vibrante.

12. Les Simpsons ne pouvaient négliger cette offre succulente consistant à faire le remake du générique de Mad Men, qui contient nombre de pulsions présentes dans le célèbre cartoon. En outre, la chute de Draper sur le divan semble déjà une citation du générique de la série consacrée à la famille américaine dessinée par Matt Groening. 


\section{Bibliographie}

Attimonelli C., D'Ottavio A., To Be Continued. Il destino del corpo nei serial televisivi, CaratteriMobili, Bari, 2011.

Barthes R., Système de la mode, Éditions du Seuil, Paris, 1967.

Cicolani F., Teoria e tecnica della sigla televisiva, il paratesto televisivo e la sua applicazione pratica, Tesi di laurea, 2005.

Dorfles G. Nuovi riti, nuovi miti (1965), Einaudi, Torino, 1977.

Dusi N., La variante interna. Modularità e ripetizione nell'epitesto audiovisivo, in Mutazioni audiovisive, ETS, 2004.

Eco U., Travels in Hyperreality, Harcourt, New York, 1986.

Spaziante L., TV sui generis: strategie di presa di distanza e generi in Twin Peaks, in G. Grignaffini, M. P. Pozzato (dir.), Mondi seriali. Percorsi semiotici nella fiction, Cologno Monzese, Collana Link, RTI-Reti Televisive It, 2008.

\section{Soundtrack}

A perfect lie, G\&D remix, The Engine Room, Nettwerk Records, 2004.

Star Trek - The Next Generation, A. Courage, Gnp records, 2000.

Twin Peaks, A. Badalamenti, Warner Bros, 1991.

Woke up this morning, Chosen one mix, Alabama 3, One Little Indian, 1997. 